Supporting Information

\title{
Amine-cured glycidyl esters as dual dynamic epoxy vitrimers
}

Yingchun Sun, ${ }^{\ddagger}$ Miaomiao Wang, ${ }^{\ddagger}$ Zhen Wang, Yifan Mao, Ling Jin, Kui Zhang, Youyi Xia and Hong Gao*

School of Chemistry and Chemical Engineering, Anhui University of Technology, Ma'anshan Anhui 243032, China. 
Table S1. The formula of xSE-yTE-SS

\begin{tabular}{ccccc}
\hline Samples codes & SE(g) & TE $(\mathrm{g})$ & SS(g) & $\begin{array}{c}\text { TBD }(\mathrm{g}) \\
(5 \mathrm{~mol} \%-\mathrm{COO} \text { of TE } \\
\text { and SE })\end{array}$ \\
\hline 10SE-0TE-SS & 10 & 0 & 3.9554 & 0.4433 \\
9SE-1TE-SS & 9 & 1 & 4.0066 & 0.4491 \\
8SE-2TE-SS & 8 & 2 & 4.0578 & 0.4547 \\
7SE-3TE-SS & 7 & 3 & 4.1091 & 0.4605 \\
6SE-4TE-SS & 6 & 4 & 4.1602 & 0.4663 \\
\hline
\end{tabular}

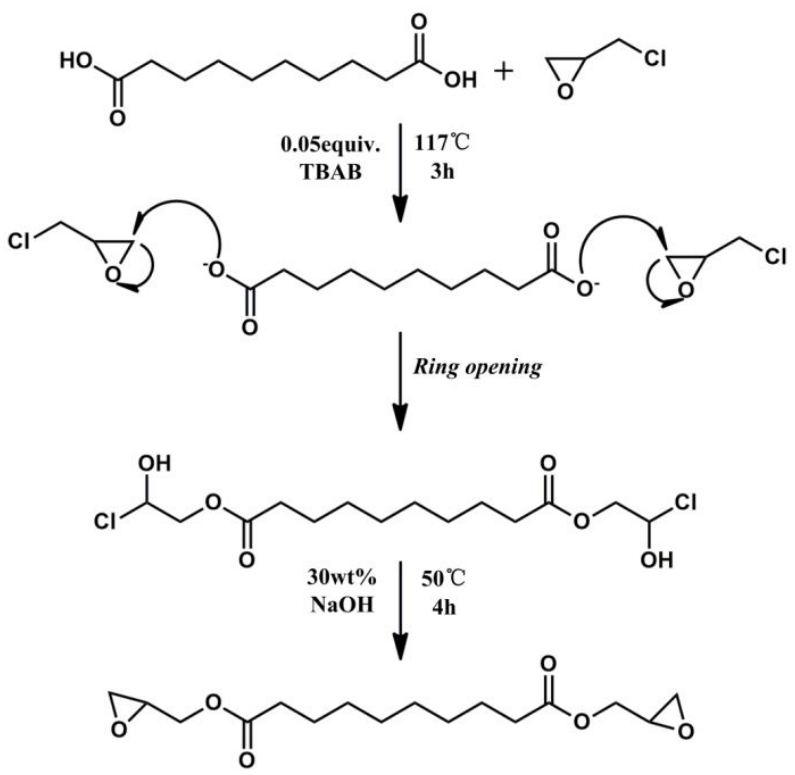

Scheme S1. Reaction mechanism of the glycidyl ester by the reaction of carboxylic acid with $\mathrm{ECH}$. 

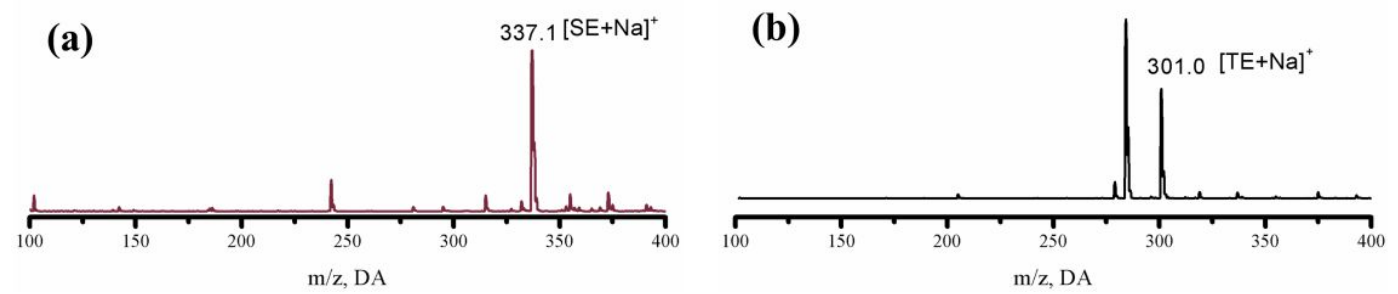

Figure S1. Mass spectrometry of SE (a) and TE (b).
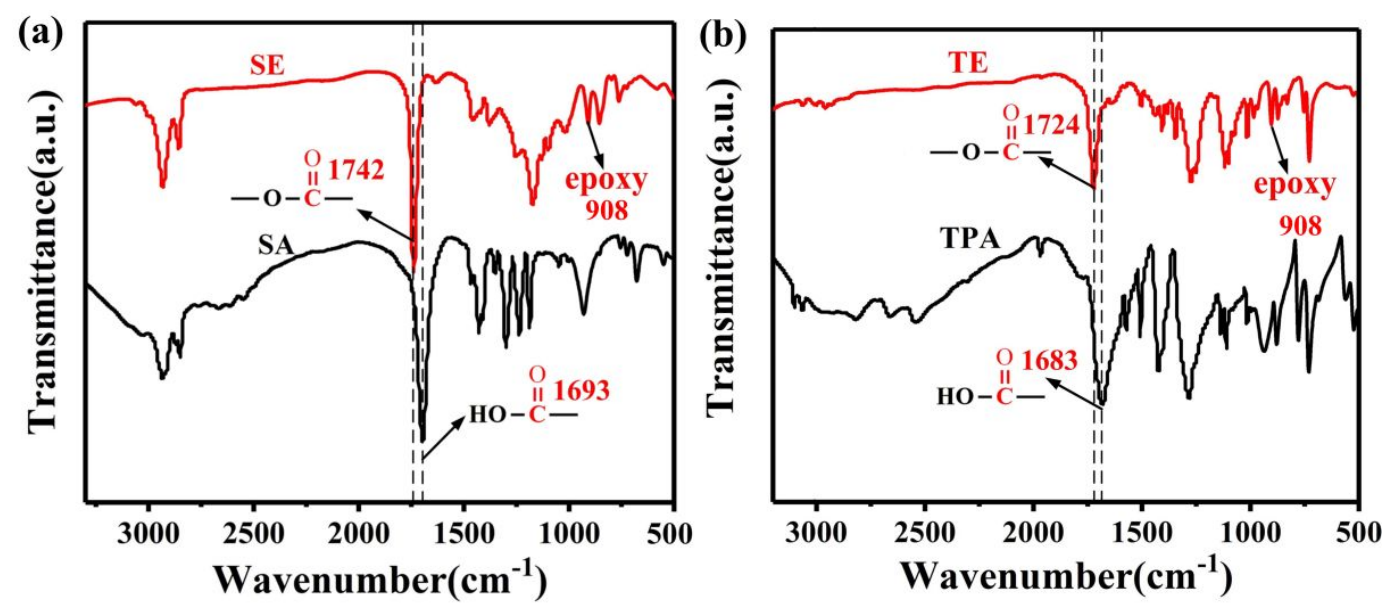

Figure S2. (a)FTIR spectra of sebacic acid (SA) and its epoxide (SE). (b) FTIR spectra of terephthalic acid (TPA) and its epoxide (TE).
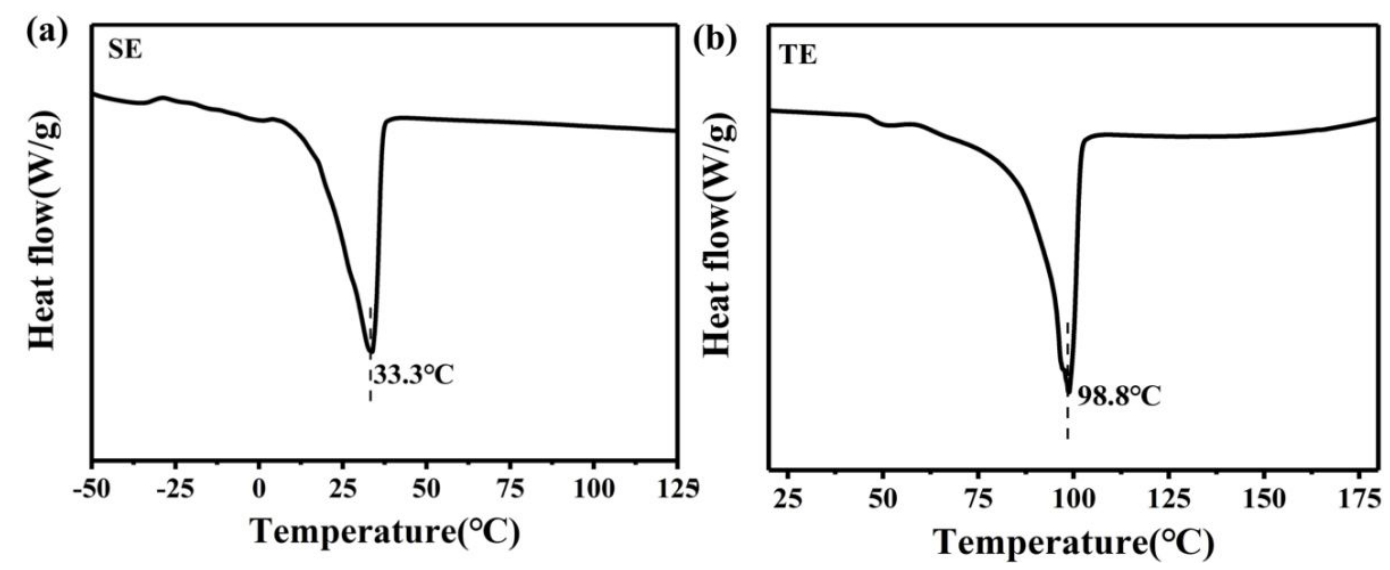

Figure S3. DSC curves of SE(a) and TE(b). 

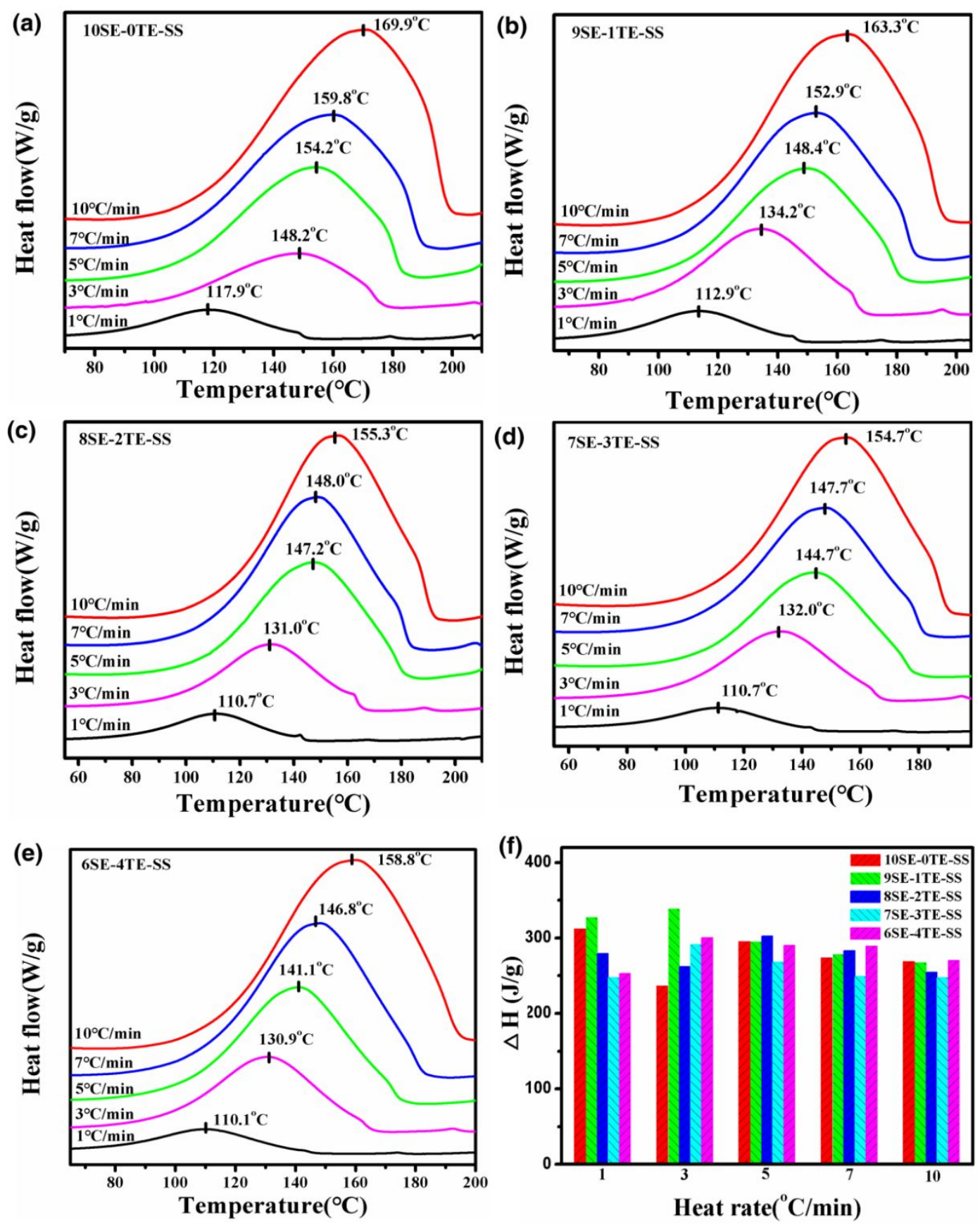

Figure S4. DSC thermograms of non-isothermal curing of (a) 10SE-0TE-SS, (b) 9SE-1TE-SS, (c) 8SE-2TE-SS, (d) 7SE-3TE-SS and (e) 6SE-4TE-SS. (f) The curing enthalpy of xSE-yTE-SS at different heating rates. 

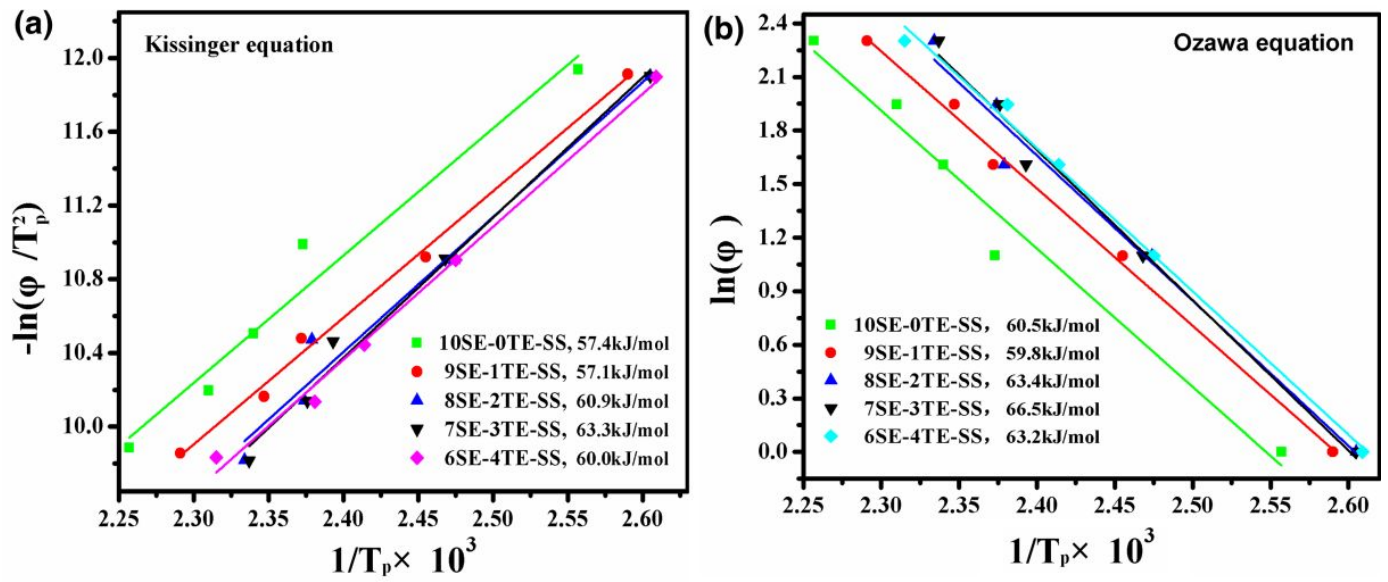

Figure S5. The activation energy $\left(E_{a}\right)$ of curing. (a) Fitting curves based on Kissinger equation. (b) Fitting curves based on Ozawa equation.

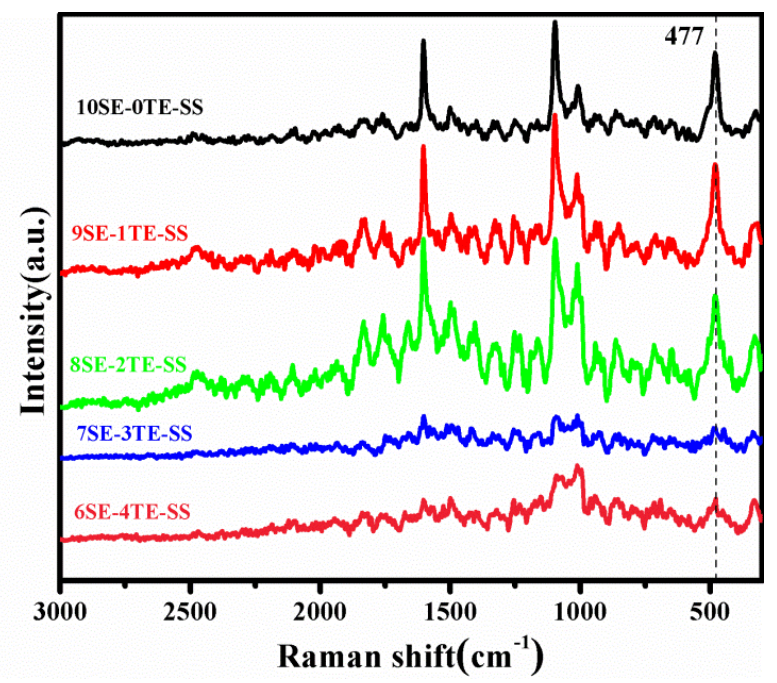

Figure S6. Raman spectra of xSE-yTE-SS. 


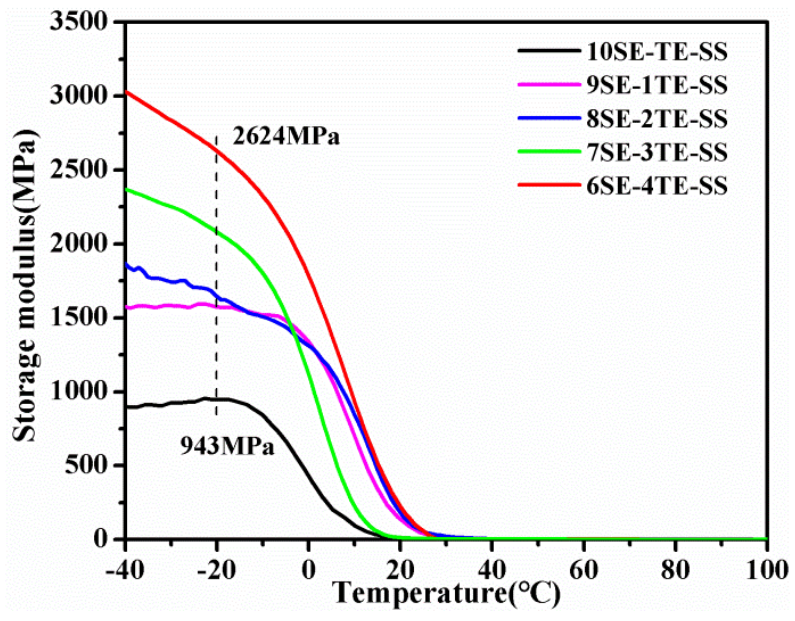

Figure S7. Storage modulus of xSE-yTE-SS.

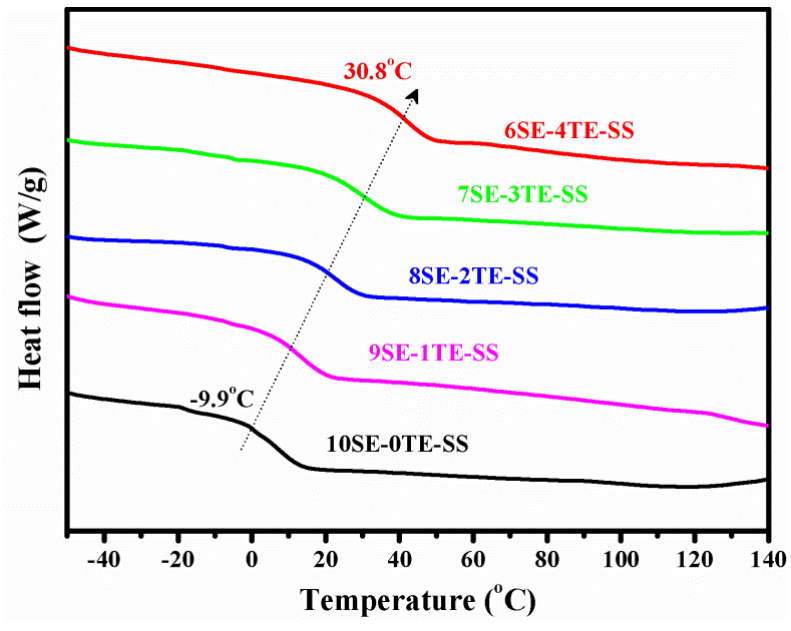

Figure S8. DSC curves of xSE-yTE-SS. 


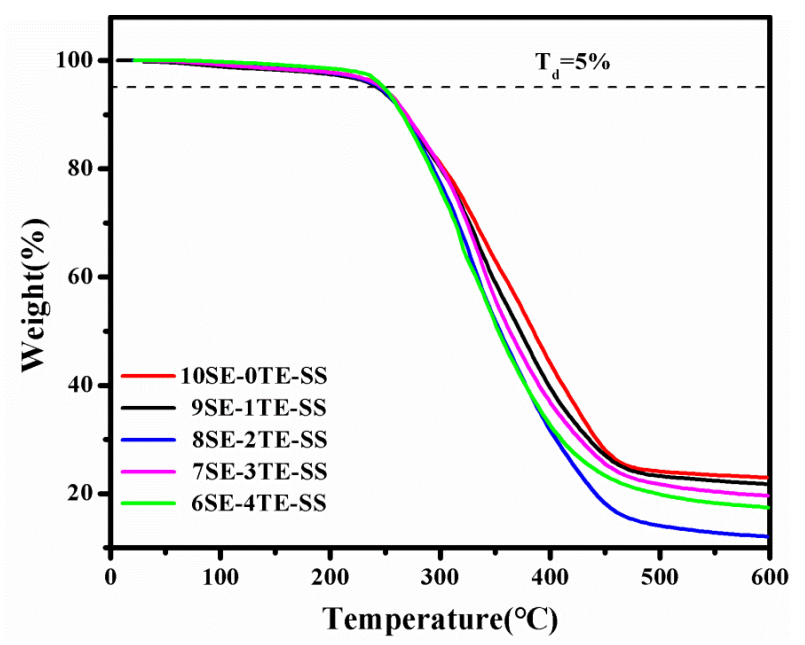

Figure S9. TGA curves of xSE-yTE-SS.

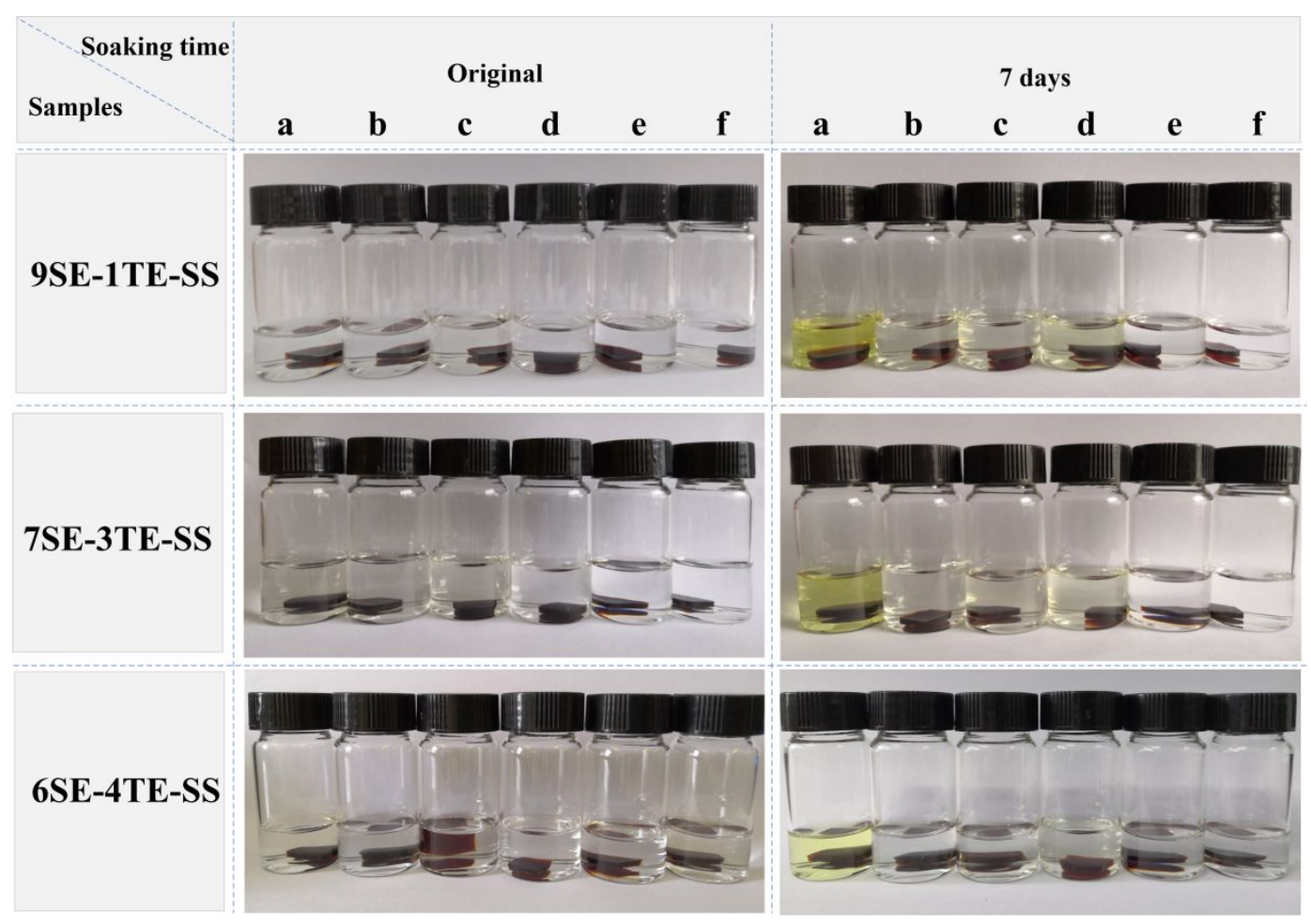

Figure S10. The chemical resistances of 9SE-1TE-SS, 7SE-3TE-SS and 6SE-4TE-SS in different solvents (a. methanol, b. isopropanol, c. triethylamine, d. acetate ethyl, e. toluene, and f. petroleum ether in order of polarity from large to small) at $25^{\circ} \mathrm{C}$ before and after 7 days. 

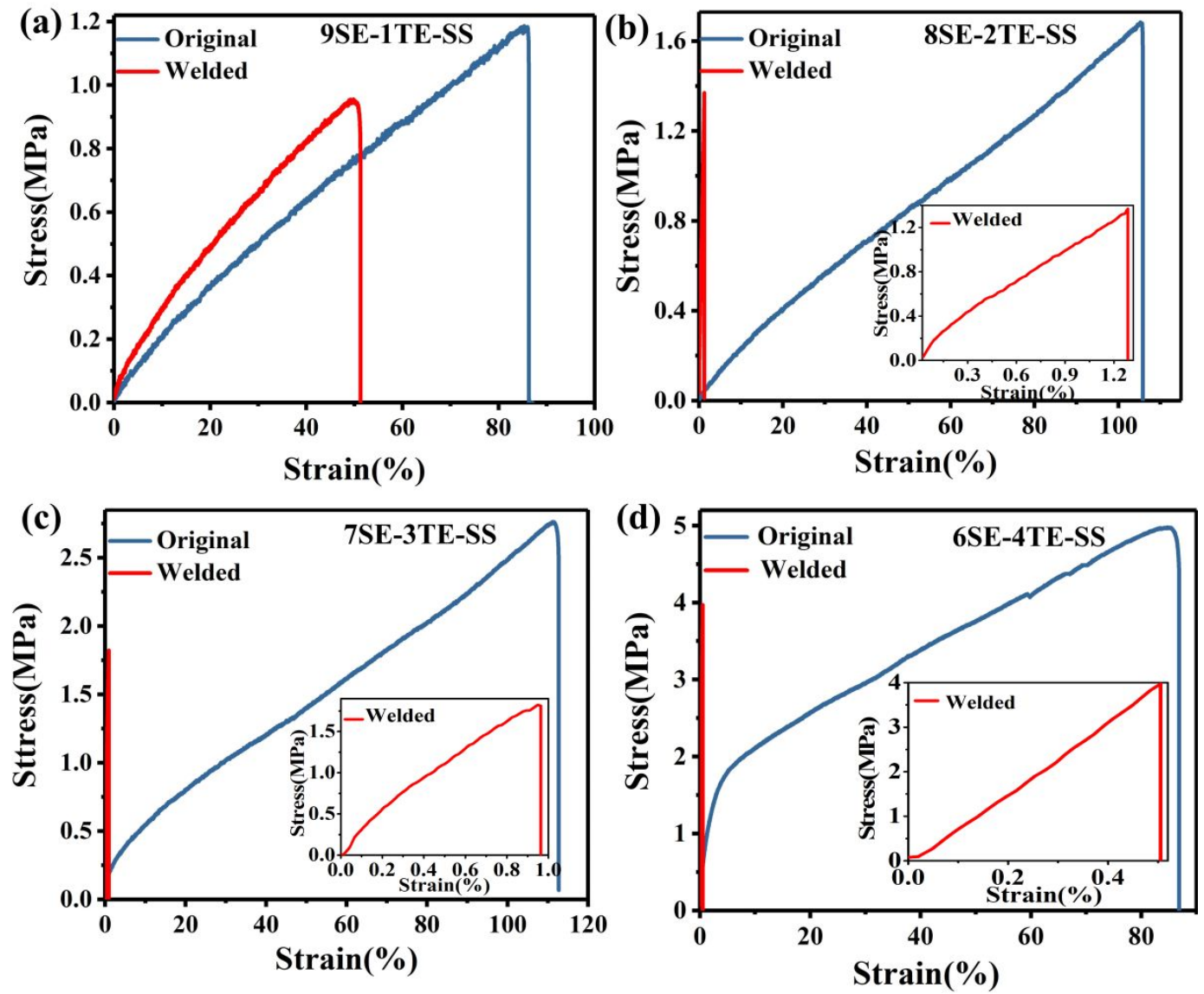

Figure S11. The stress-strain curves of (a) 9SE-1TE-SS, (b) 8SE-2TE-SS, (c) 7SE-3TE-SS and (d) 6SE-4TE-SS before and after welding. The inset ones are the amplifying stress-strain curves after welding. 


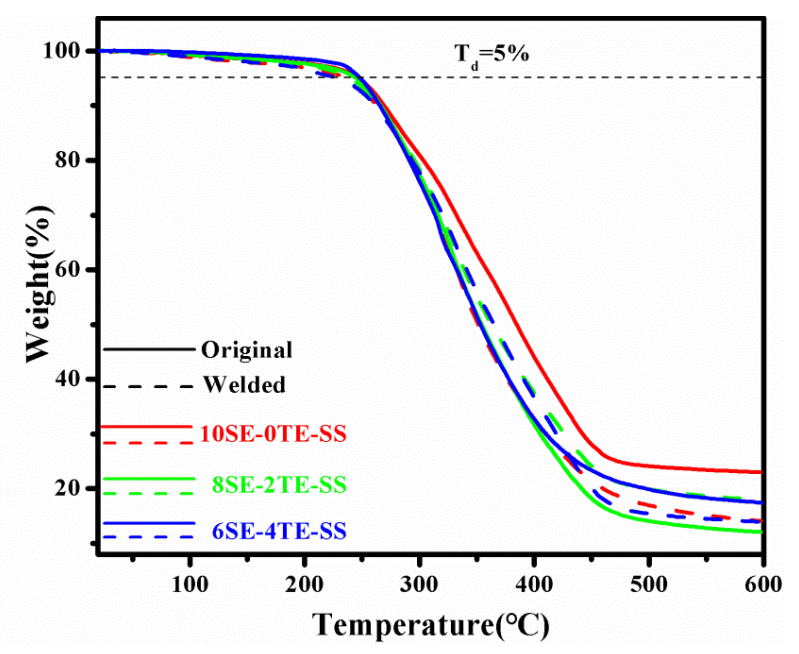

Figure S12. TGA curves of xSE-yTE-SS before and after welding.

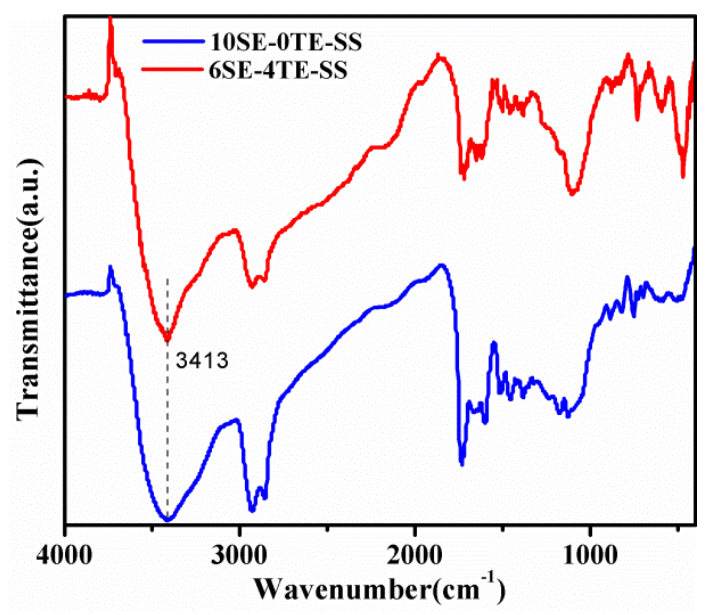

Figure S13. FTIR spectra of the recycled 6SE-4TE-SS and 10SE-TE-SS. 


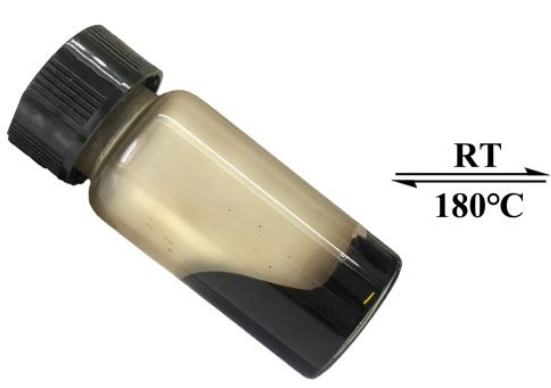

Flowing

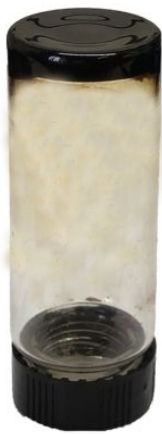

Freezing

Figure S14. The state transition images of the recycled dual dynamic vitrimers at $180^{\circ} \mathrm{C}$ and room temperature.

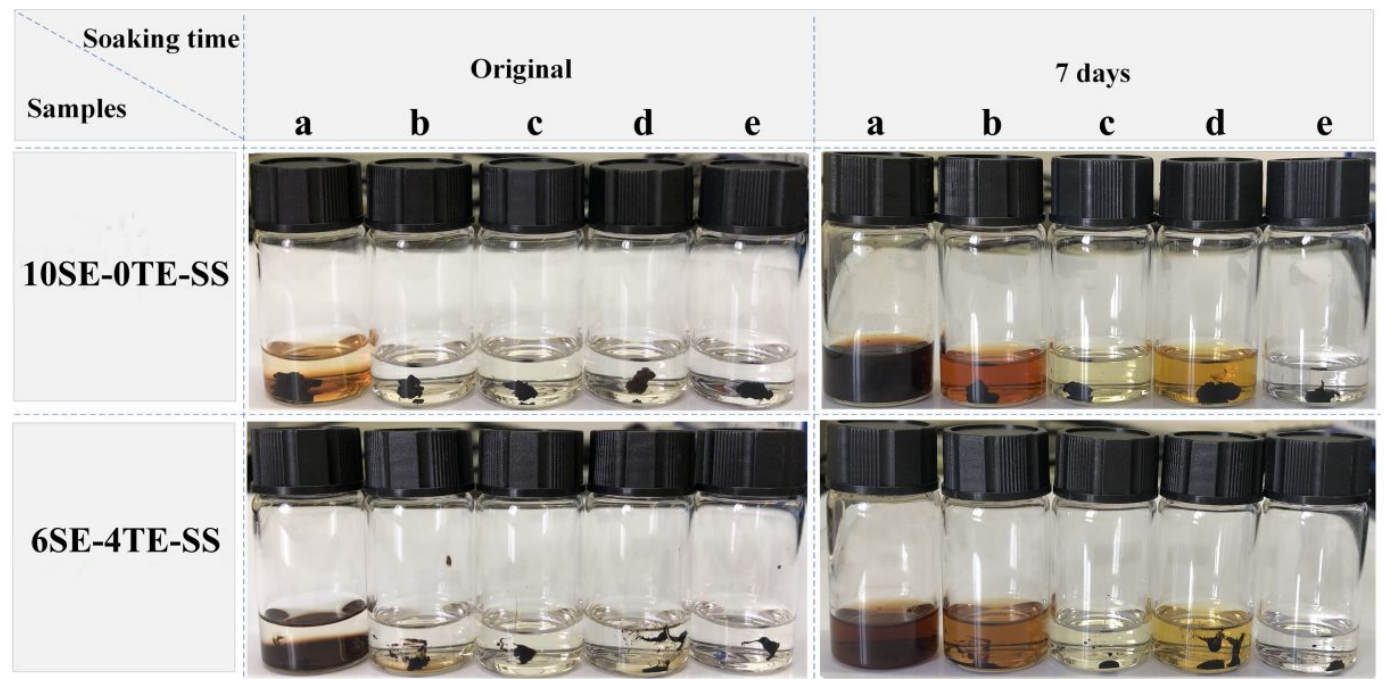

Figure S15. The chemical resistances of the recycled 10SE-0TE-SS, and 6SE-4TE-SS in different solvents (a. methanol, b. isopropanol, c. triethylamine, d. acetate ethyl, and e. toluene in order of polarity from large to small) at $25^{\circ} \mathrm{C}$ before and after 7 days. 


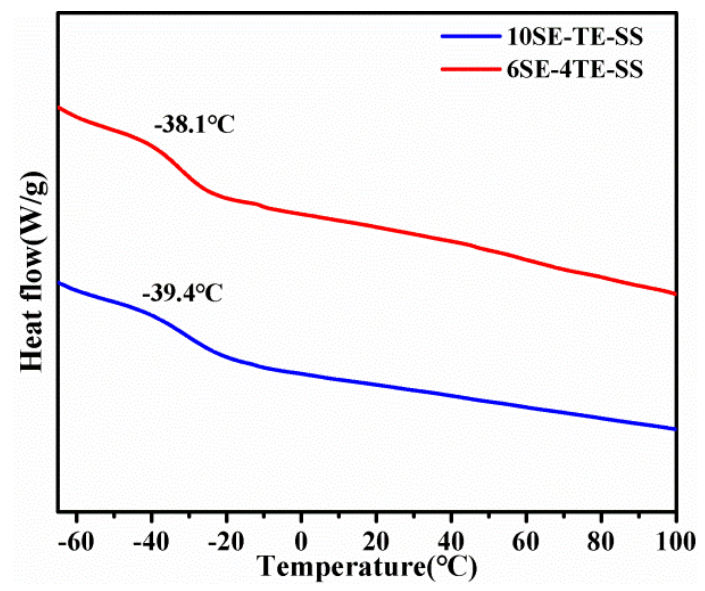

Figure S16. DSC curves of the recycled 10SE-0TE-SS and 6SE-4TE-SS.

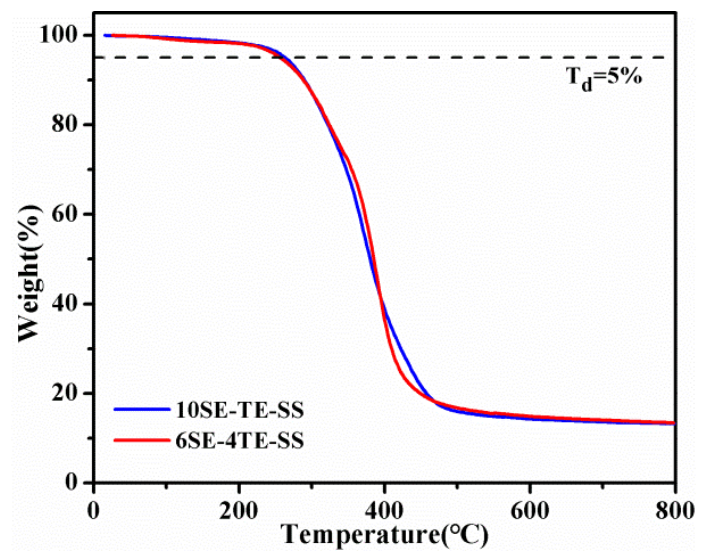

Figure S17. TGA curves of the recycled 10SE-0TE-SS and 6SE-4TE-SS. 

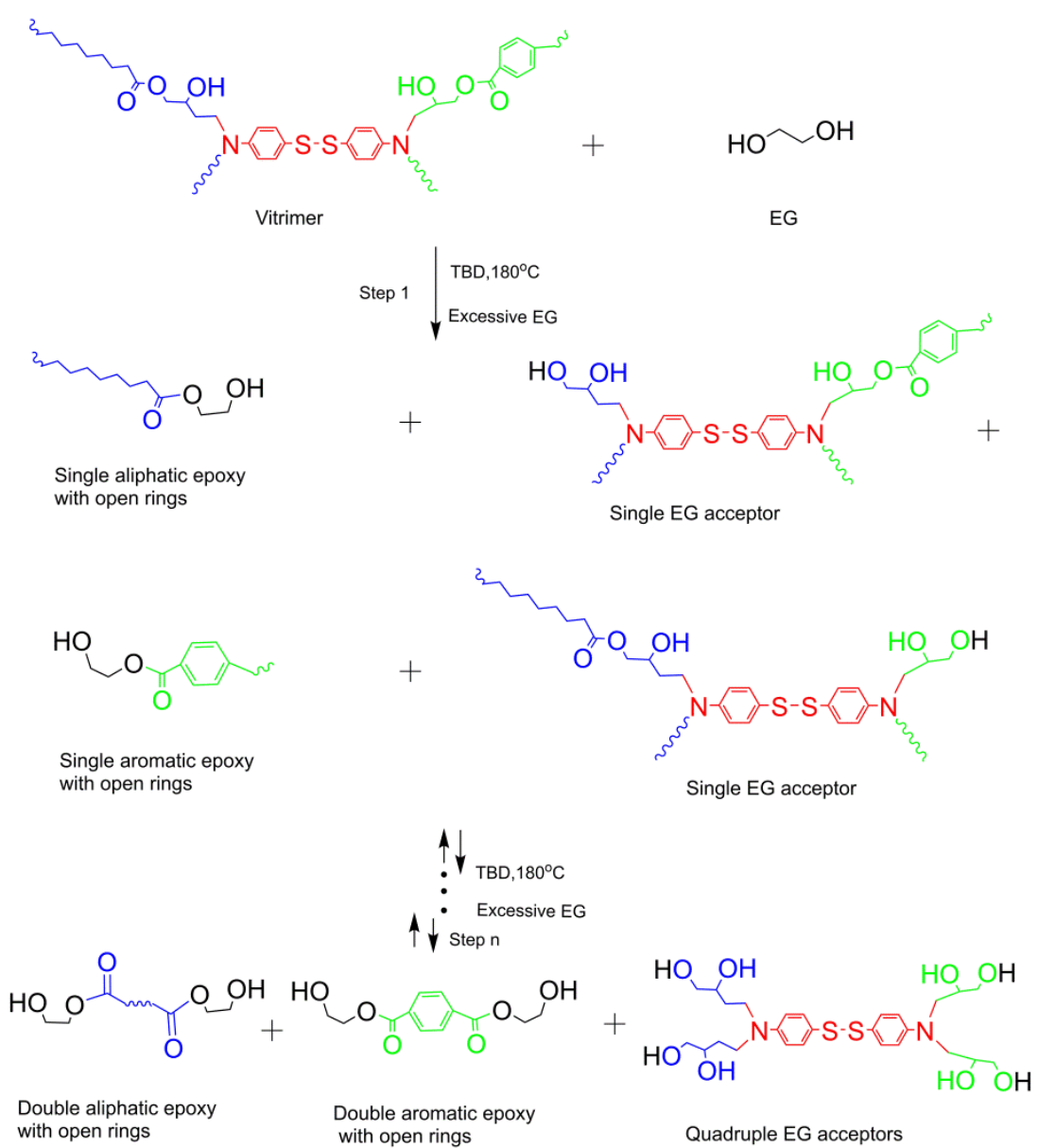

Figure S18. Hypothetical chemical reactions during crosslinking/dissolution cycles of the recyclable vitrimers. Step1: EG molecules participate in the polymer networks at the ester sites to break them. Step2: the balance between depolymerized chains and repolymerization together at the same ester sites. 


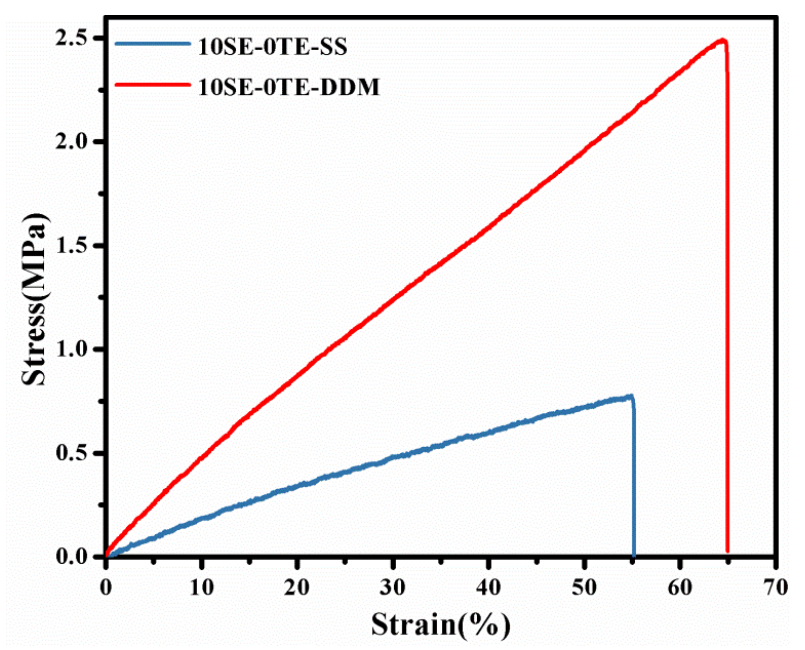

Figure S19. Representative stress-strain curves for 10SE-0TE-SS and 10SE-TE-DDM.
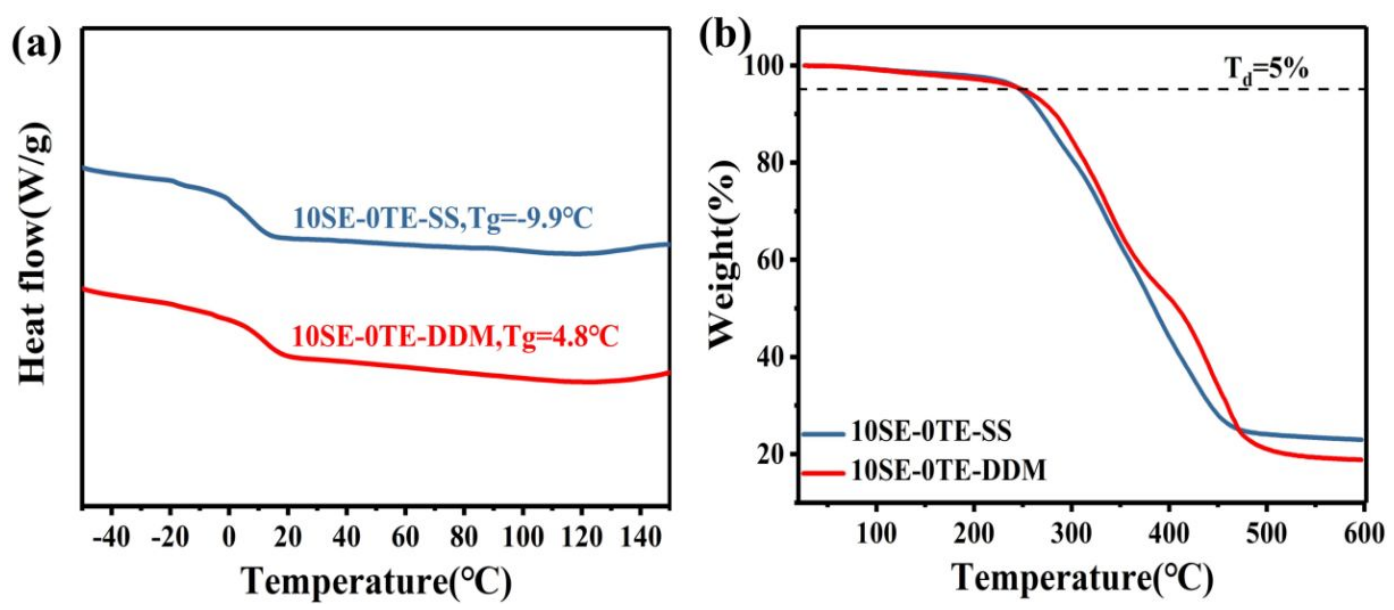

Figure S20. (a) DSC and (b) TGA curves of 10SE-0TE-SS and 10SE-0TE-DDM. 

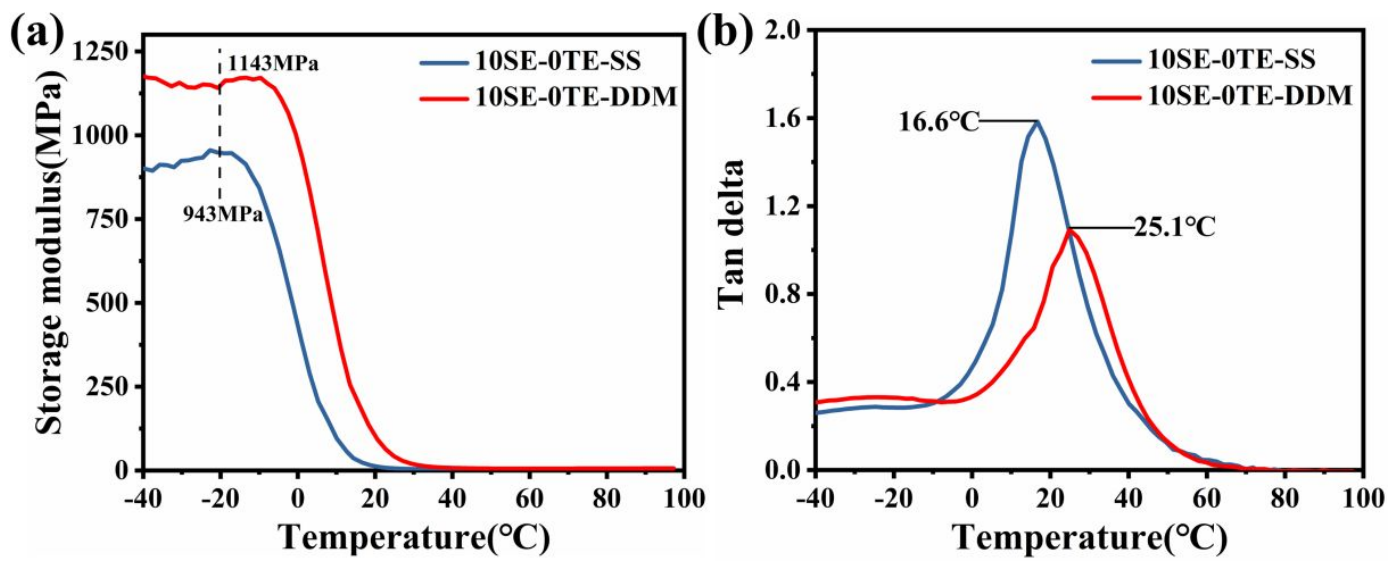

Figure S21. (a)Storage modulus and (b) tan delta curves of 10SE-0TE-SS and 10SE-0TE-DDM.

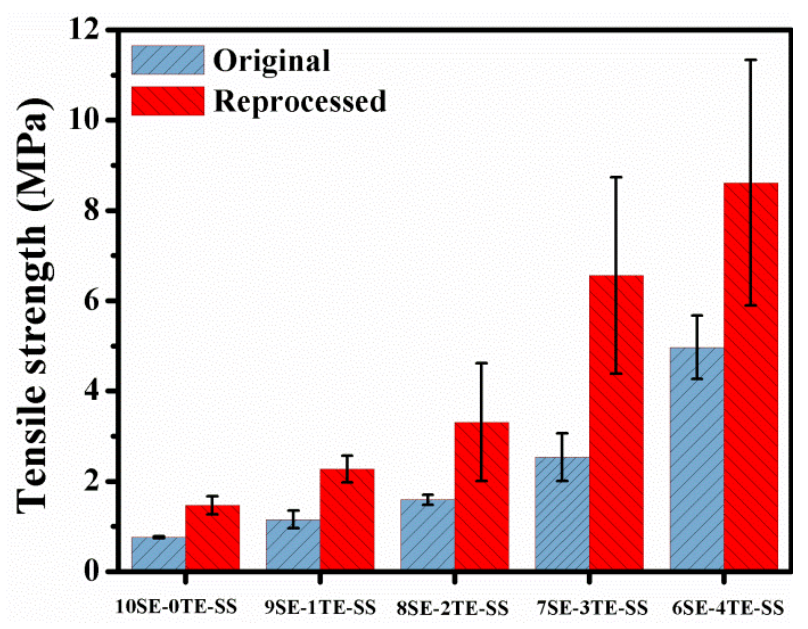

Figure S22. The tensile strengths of dual dynamic vitrimers before and after reprocessing. 


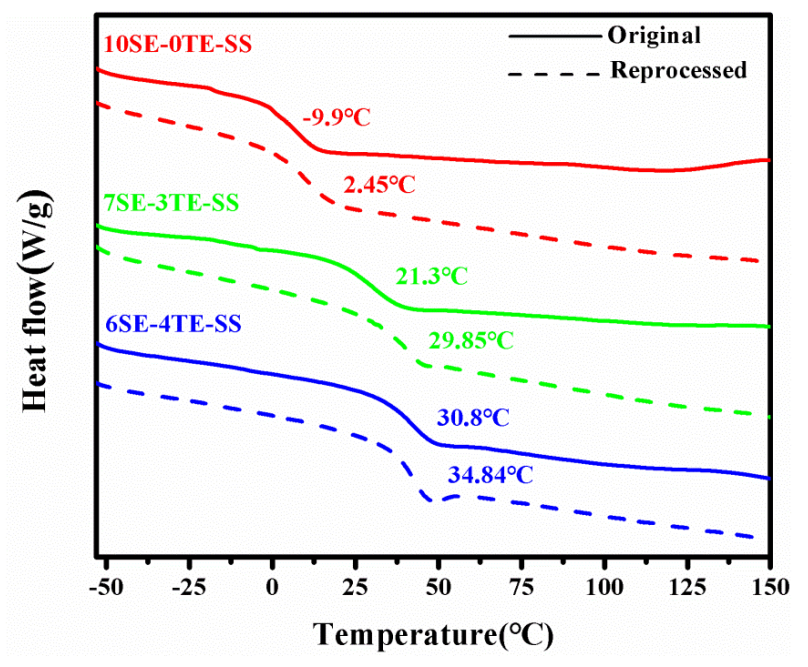

Figure S23. DSC curves of xSE-yTE-SS before and after reprocessing.

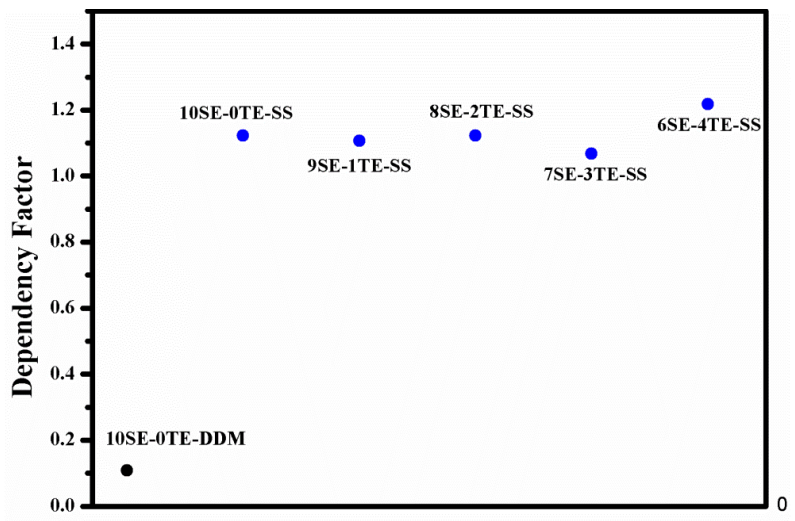

Figure S24. Dependency factor/index (x) was calculated using by fitting the $\mathrm{G}^{\prime}$ data in Figure 5c and fitting it into the power law relationship $\mathrm{G}^{\prime} \sim \omega^{\mathrm{x}}$, where $\mathrm{x}=0$ for a perfect polymer network. $\mathrm{G}^{\prime}$ value of a perfect crosslinked network is steady without depending on frequency and any dependency is often attributed to an 'imperfect' network structure. 


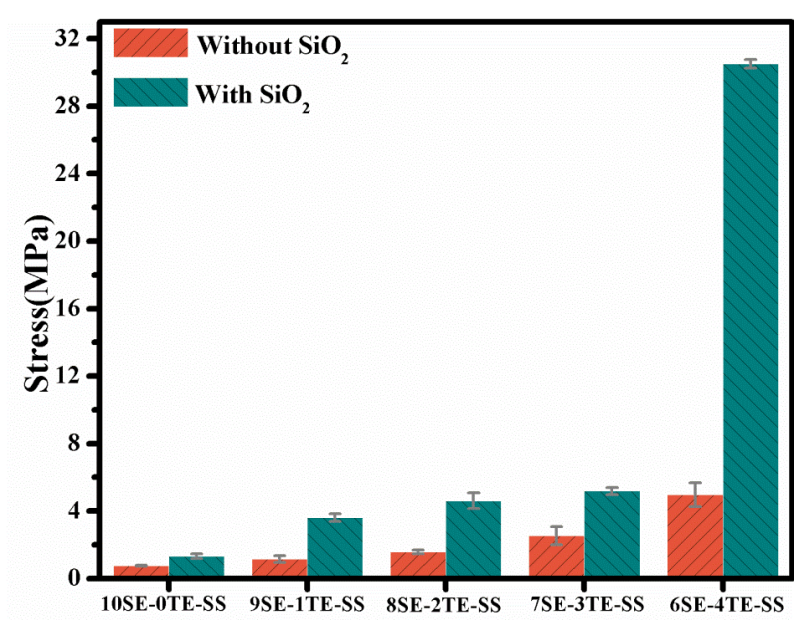

Figure S25. Average strength for xSE-yTE-SS before and after adding $\mathrm{SiO}_{2}$.

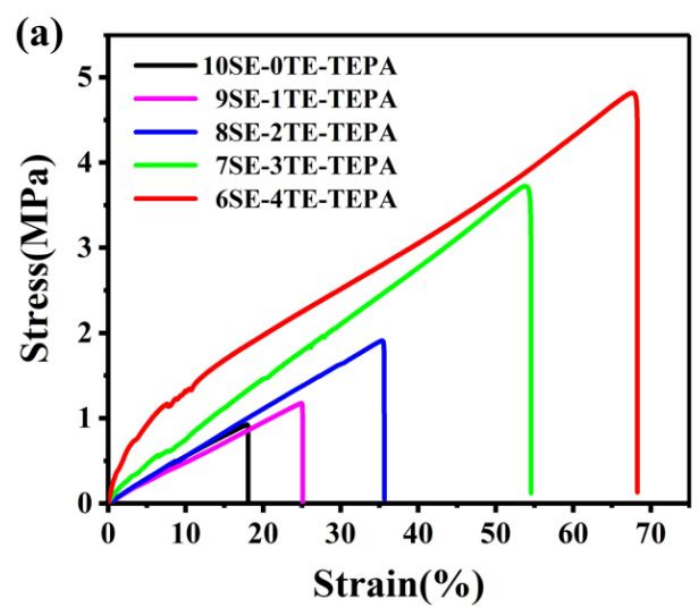

(b)

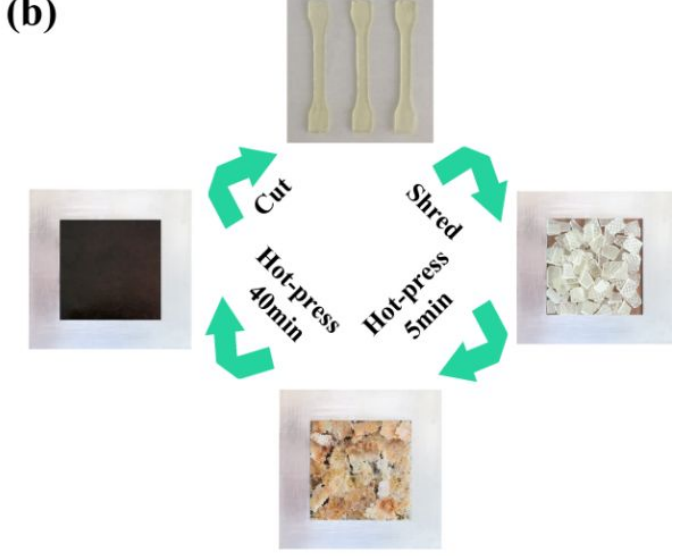

Figure S26. (a) Representative tensile stress-strain curves of xSE-yTE-TEPA. (b) xSE-yTE-TEPA at $180{ }^{\circ} \mathrm{C}$ for $40 \mathrm{~min}$ to get a reprocessed sample. 